\title{
A Simplified Technique for Drainage of Chronic Calcified Pleural Empyema
}

\author{
Sani Rabiou ${ }^{1}$ Hama Younssa ${ }^{2}$ James Didier ${ }^{2}$ Magagi Ibrahim ${ }^{3}$ Adamou Harissou ${ }^{3}$ Ousseini Adakal ${ }^{4}$ \\ Rachid Sani ${ }^{2}$
}

${ }^{1}$ Department of Thoracic Surgery, CHU Hassan II,

Fes, Morocco

2 Department of General Surgery, HNN, Niamey, Niger

3 Department of General Surgery, HNZ, Zinder, Niger

${ }^{4}$ Department of General Surgery, HRM, Maradi, Niger

Thorac Cardiovasc Surg 2017;65:586-588.

Address for correspondence Sani Rabiou, Department of Thoracic Surgery, CHU Hassan II, Fes, Morocco (e-mail: rabiousani2@yahoo.fr).

\begin{abstract}
Keywords

- tuberculosis

- thoracic empyema

- pyothorax

- thoracostomy

Chronic pleural pocket has well-known diagnosis and treatment principles since first described by Hippocrates 2,400 years ago. However, its treatment remains constant even though its causes, severity during management, and terrain vary considerably. In well-structured health care systems, posttuberculous empyema has become rare; its well-codified medical treatment relies on early diagnosis and adapted antibiotherapy, punctures/drainage, and appropriate intrapleural antifibrinolytics. In developing countries, a poor health organizational system increases the incidence of pleural pocket, which can progress until surgery is indicated. In such a context, the general principles of treatment include pleural decortication along with pulmonary resection. This technique remains difficult, risky, and, sometimes, impossible due to the chronicity of the lesion. In patients debilitated by several months of septic evolution, a simplified thoracostomy technique permits complete resection of the pocket.
\end{abstract}

\section{Introduction}

In the 1980s, the incidence of tuberculosis peaked especially in low-income countries with increased resistance of the Koch bacilli to antituberculous drugs. This phenomenon is related to the HIV-AIDS (human immunodeficiency virus infection-acquired immune deficiency syndrome) pandemic, population migration, poor living conditions (poverty, joblessness, drug addiction), and problems due to treatment compliance. ${ }^{1}$ If developed countries offer complete treatment for early diagnosed cases, management of complicated forms of tuberculosis seen in developing countries such as chronic pyothorax with calcified pleural pockets seems risky or impossible. In such conditions, open thoracostomy in treatment in cases of pleural empyema with chronic pyothorax appears to be a possible option. The original Schede operation (Schede thoracostomy) was an intervention that aimed at

received

September 23, 2016

accepted

November 6, 2016

published online

January 28, 2017

evacuating pleural pockets fused with the thoracic wall. It consists of large resection of the thoracic wall, ribs, intercostal muscles, and parietal pleura, leaving a U-shaped skin flap to drain pleural collection and continuous dressing to obtain detersion and scarification. The original technique involved an extended parietal resection and prolonged dressing. The modern thoracoplasty involves only subperiosteal resection of several ribs; by removing the skeletal support, the overlying chest wall will collapse to the visceral pleura to obliterate the empyema cavity. Hence, the indications of an open drainage in pleural collections include chronic tuberculous or nontuberculous pyothorax and pleural pocket after surgical pulmonary resections. Drainage by Delbet drain ( - Fig. 1) that passes around an intercostal space after resection of two costal segments can be an alternative to conventional thoracostomy. This technique is advantageous in simplifying and

(c) 2017 Georg Thieme Verlag KG Stuttgart · New York
DOI https://doi.org/ 10.1055/s-0036-1597592. ISSN 0171-6425. 
reducing dressing and postoperative postoperative care. It can also replace the conventional technique in other situations. $^{2-4}$ Because of its simple steps and less invasiveness nature, we propose this modified technique in the management of certain chronic calcified pleural pockets or in the treatment of pyothorax in a pneumonectomy or lobectomy cavity. The objective was to shorten the period of hospitalization, permit early discharge, and avoid complications related to pleuropulmonary decortication.

\section{Technique Description}

The patient is placed in lateral decubitus position. Incision is done over the collection, which if possible was imaged and marked before the surgery. If the incision passes across the latissimus dorsi muscle, a vertical incision along the direction of the fibers is preferred to conserve the muscle. This muscle remains vascularized by perforating branches of the intercostal arteries and can be used later on for myoplasty (-Fig. 2). After skin and muscle incision, we palpate the ribs above and below the intercostal space chosen. These ribs are carefully cleaned and exposed on elastic tapes to make their resection easy (-Fig. 3 ). The chosen rib is cut over $4 \mathrm{~cm}$, and the pleura is incised in the bed of the rib. This permits opening the pleural cavity underneath for evacuation. With digital maneuver, we choose the second rib to be resected; this could be one level above or one below depending on the pleural collection. This second rib is again resected over $4 \mathrm{~cm}$ ( $\mathbf{- F i g . ~ 4 A , ~ B ) . ~ T h e ~ p l e u r a ~ a l r e a d y ~ o p e n e d ~ i n ~ t h e ~ b e d s ~ o f ~}$ the two ribs is resected. The pleural pockets are thoroughly washed with an antiseptic solution mixed with saline

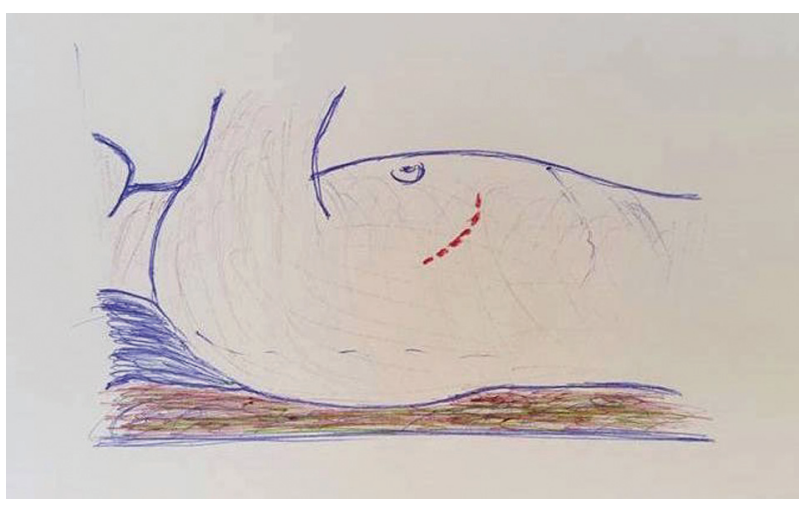

Fig. 2 Positioning and skin marking.

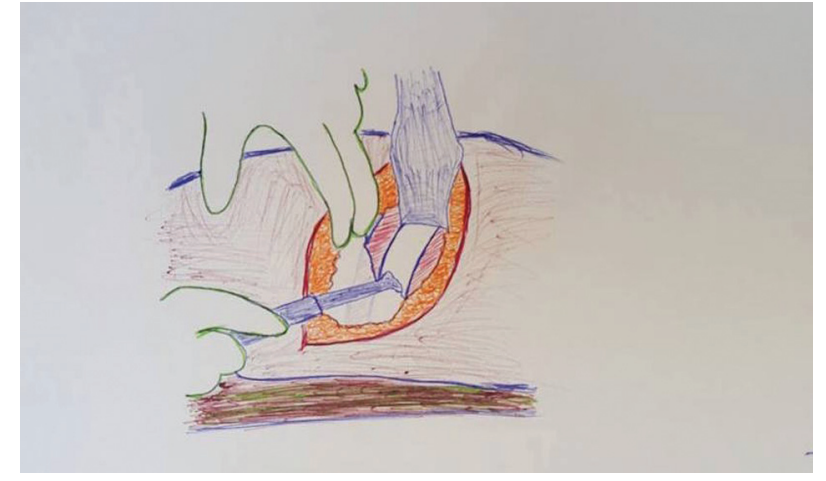

Fig. 3 Dissection and exposure of the ribs and intercostal space.

solution. We then place the Delbet drain around the intercostal space to continuously drain the collection. The ends of the drain are fixed with nonabsorbable sutures to avoid any endothoracic migration (-Fig. 5A, B). Wound dressing is done at bedside, initially daily and later every 2 days. The follow-up is clinical and by imaging. A computed tomographic (CT) scan of the chest and bronchoscopy are helpful in the presence of concomitant pleural pockets and bronchial fistula. The patient is discharged if the general state improves; however, dressings should continue at home. Generally after 2 weeks to 3 months, a control CT scan is performed to demonstrate the disappearance of the pleural cavity. We then remove the Delbet drain and the draining orifice closes some days later. In cases of persistence of the pleural cavity, presence of a bronchial fistula, or nonclosure of the orifice after removal of the Delbet drain, the patient is referred later for a myoplasty to accelerate healing. ${ }^{4}$

\section{Results}

Fourteen patients were enrolled in this study from January 2004 to December 2014. ${ }^{4}$ These patients included six cases of pleural pockets after pulmonary resection and three cases of chronic purulent pleurisy drained after failure of pulmonary reexpansion. The five other cases involved pulmonary infection with complications of empyema. In four of these patients, this technique was done secondary to complicated pulmonary resection or after initial pleural drainage. After a mean 56 days of follow-up, no new infection was noted and the primary infection was controlled. Other authors ${ }^{5}$ proposed a technique similar to ours. It consists of placing a tubular prosthesis in the bed of the resected rib to maintain the open drainage pathway. The principle is globally the same as the Delbet drain with similar results.

\section{Conclusion}

The technique we propose simplifies conventional open thoracostomy indicated for patients with chronic calcified pleural pockets. It can be used as a definitive treatment but also as an alternative prior to myoplasty. With satisfactory 


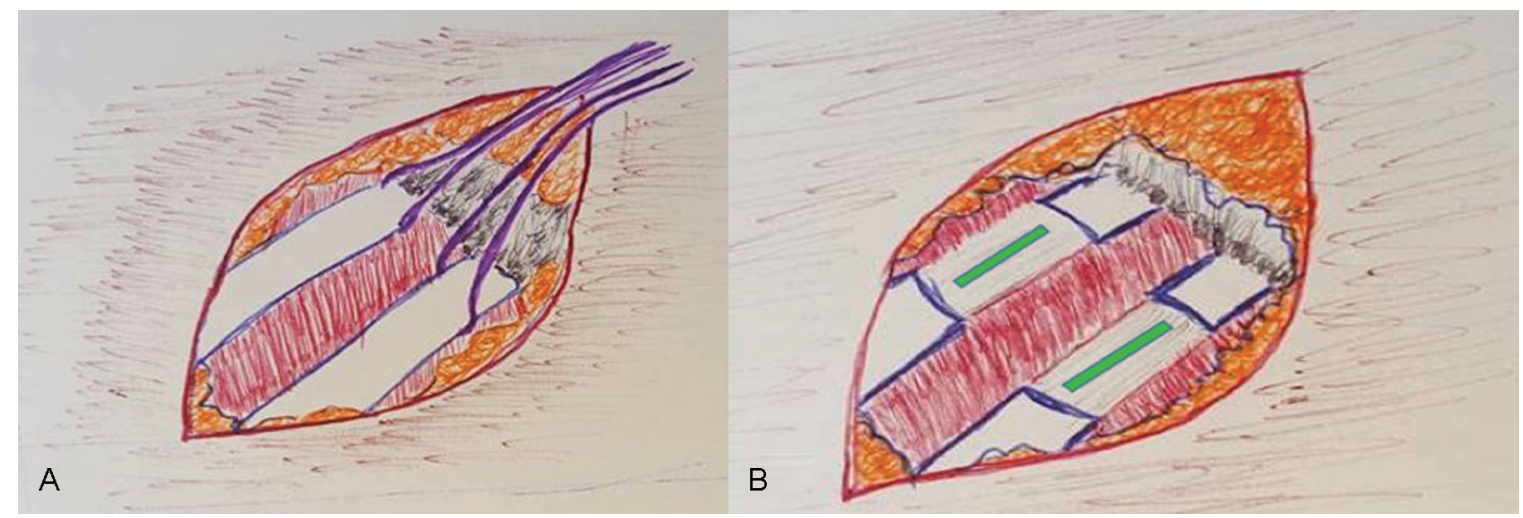

Fig. 4 (A, B) Resection of the ribs and opening of the pleura.
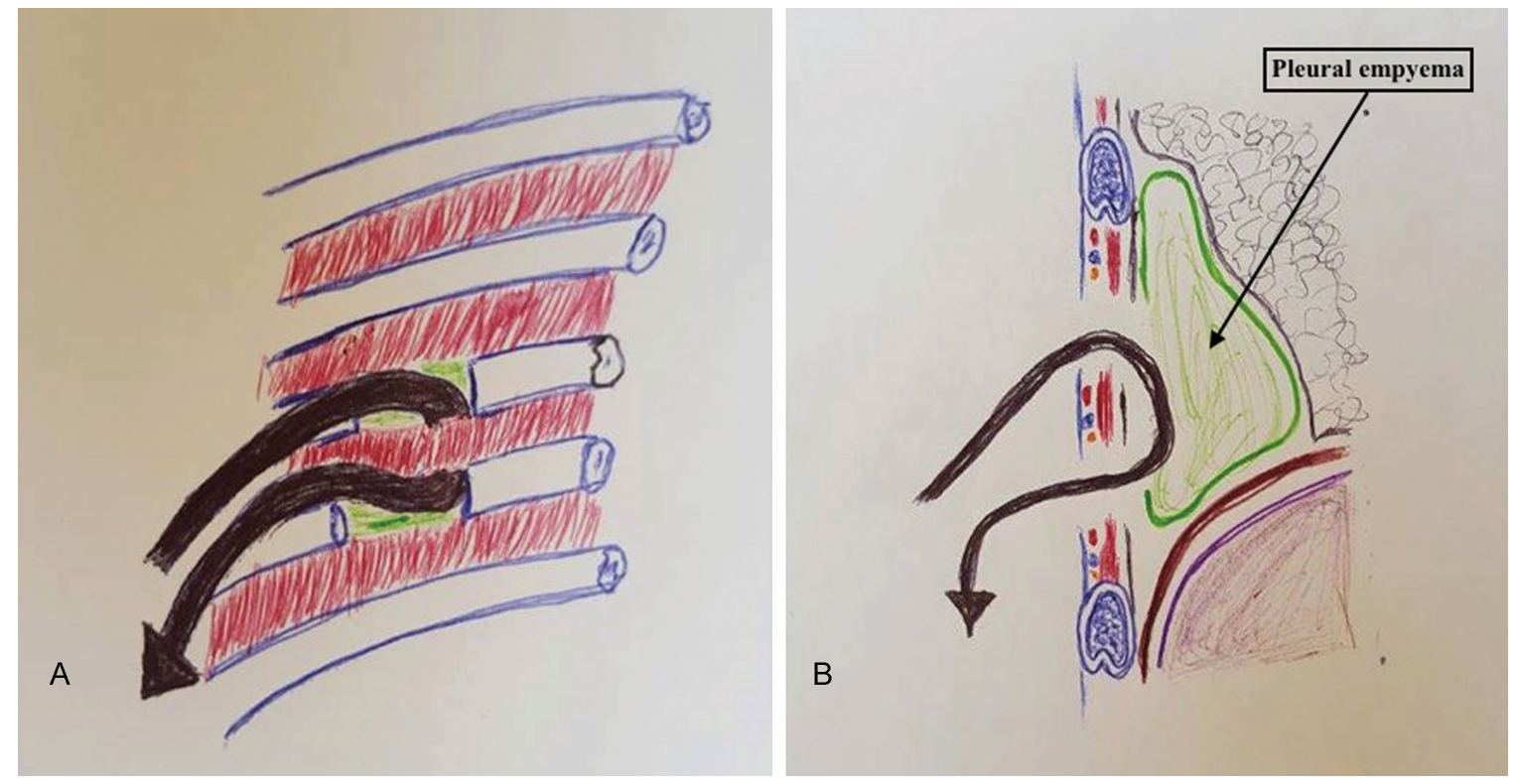

Fig. 5 (A, B) Placement of the Delbet drain.

results, it limits all constraints related to nursing and also reduces hospital duration.

\section{Conflicts of Interest}

None.

\section{References}

1 Rosenblatt MB. Pulmonary tuberculosis: evolution of modern therapy. Bull N Y Acad Med 1973;49(3):163-196
2 Deslauriers J, Jacques LF, Grégoire J. Role of Eloesser flap and thoracoplasty in the third millennium. Chest Surg Clin N Am 2002; 12(3):605-623

3 Hertzog P, Toty L, Personne C, Riquet M. [Repair of long-term cavernostomies and parieto-pleurectomies using myoplasty and cutaneous autoplasty]. Ann Chir 1980;34(8):631-635

4 Bellamy J, Onea F. Une technique simplifiée de pleurostomie. Chir Thor Cardiovasc 2011;15:56-59

5 Filomeno LT, Campos JR, Machuca TN, das Neves-Pereira JC, Terra RM. Prosthesis for open pleurostomy (POP): management for chronic empyemas. Clinics (Sao Paulo) 2009;64(3): 203-208 